\title{
Uremic Plasma Impairs Barrier Function and Depletes the Tight Junction Protein Constituents of Intestinal Epithelium
}

\author{
Nosratola D. Vaziria Nisa Goshtasbi ${ }^{a}$ Jun Yuan ${ }^{a}$ Stefan Jellbauer ${ }^{b}$ \\ Hamid Moradi $^{a}$ Manuela Raffatellu $^{\mathrm{b}}$ Kamyar Kalantar-Zadeh ${ }^{\mathrm{a}}$ \\ aDivision of Nephrology and Hypertension, and ${ }^{b}$ Department of Microbiology and Molecular Genetics, \\ University of California, Irvine, Calif., USA
}

\section{Key Words}

Tight junction $\cdot$ Inflammation $\cdot$ Hemodialysis $\cdot$ End-stage renal disease $\cdot$ Chronic kidney disease

\begin{abstract}
Background: Chronic kidney disease (CKD) causes intestinal barrier dysfunction which by allowing influx of endotoxin and other noxious products contributes to the CKD-associated systemic inflammation and uremic toxicity. We have recently shown that intestinal barrier dysfunction in CKD animals is due to degradation of transcellular (claudin-1 and occludin) and intracellular (ZO1) constituents of epithelial tight junction (TJ). This study determined whether CKD-associated disruption of $\mathrm{T} J$ is mediated by retained uremic toxins/metabolites and, if so, whether they are removed by hemodialysis. Methods: The TJ-forming human enterocytes (T84 cells) were seeded on the Transwell plates and utilized when transepithelial electrical resistance (TER) exceeded $1,000 \mathrm{~m} \Omega / \mathrm{cm}^{2}$ to ensure full polarization and TJ formation. The cells were then incubated for $24 \mathrm{~h}$ in media containing $10 \%$ pre- or posthemodialysis plasma from end-stage renal disease (ESRD) patients or healthy individuals. TER was then measured and cells were processed for Western blot and immunohistological analyses. Results: Compared with the control plasma, incubation in media containing predialysis
\end{abstract}

plasma from ESRD patients resulted in a marked drop in TER pointing to increased epithelial permeability. This was accompanied by significant reductions in claudin-1 (85\%), occludin (15\%), and ZO1 (70\%) abundance. The severity of TJ damage and dysfunction was significantly less in cells exposed to the postdialysis in comparison to predialysis plasma. These findings point to the presence of as-yet unidentified product(s) in the uremic plasma capable of depleting epithelial TJ. Conclusions: Exposure to uremic milieu damages the intestinal epithelial TJ and impairs its barrier function, events which are mediated by agents which are partially removed by hemodialysis.

Copyright $\odot 2012$ S. Karger AG, Basel

\section{Introduction}

Inflammation is a common feature and a major mediator of progression of chronic kidney disease (CKD) and its cardiovascular and numerous other complications $[1,2]$. As an extension of the external environment within the body, the gastrointestinal tract serves as a barrier against entry of the microbes, harmful microbial byproducts, and other noxious compounds into the internal environment. The intestinal barrier apparatus consists of the epithelial cells and the apical junctional complex. The

\section{KARGER}

Fax +4161306 1234

E-Mail karger@karger.ch

www.karger.com
C 2012 S. Karger AG, Basel

0250-8095/12/0365-0438\$38.00/0

Accessible online at:

www.karger.com/ajn
N.D. Vaziri, MD, MACP

Division of Nephrology and Hypertension, Suite 400, City Tower

University of California, Irvine Medical Center

101 The City Drive, Orange, CA 92868 (USA)

E-Mailndvaziri@uci.edu 
latter prevents paracellular passage of the luminal contents by sealing the gap between the adjacent epithelial cells [3]. The apical junctional complex consists of the tight junction (TJ) which is the most luminal component of the complex and the subjacent adherens junction. The TJ consists of the three components: (1) adhesive transmembrane proteins including occludin and claudin family of proteins which link the plasma membranes of the adjacent cells to form the barrier to diffusion of fluids and solutes; (2) the cytosolic proteins, i.e. the zonula occludens $(\mathrm{ZO})$ protein family which serve as the anchor by simultaneously binding the intracellular domains of occludin and claudin and the perijunctional actin-myosin ring, and (3) the perijunctional ring of actin and myosin which by modulating the structure and function of the TJ, regulate paracellular permeability [4]. The intestinal epithelial TJ forms an effective barrier against influx of microbes, microbial toxins, antigens, digestive enzymes, degraded food products, and other noxious substances from the gastrointestinal lumen to the internal milieu.

There is mounting evidence pointing to intestinal barrier dysfunction and its role in the pathogenesis of uremic toxicity and inflammation in humans and experimental animals. These include the presence of endotoxemia in uremic patients [5-8], increased intestinal permeability to large molecular weight polyethylene glycols in the uremic humans and animals $[9,10]$, penetration of bacteria across the intestinal wall and their detection in the mesenteric lymph nodes in uremic rats [11], and histological evidence of chronic inflammation throughout the gastrointestinal tract in the hemodialysis population [12]. In a recent study designed to explore the mechanism of uremia-induced intestinal barrier dysfunction, we found extensive depletion of transcellular and intracellular protein constituents of colonic epithelial TJ in rats with CKD induced by either $5 / 6$ nephrectomy or chronic tubulointerstitial nephritis [13]. These findings helped to advance our understanding of the mechanism of the previously demonstrated increased intestinal permeability in uremia. However, the mechanism by which uremia results in degradation of the intestinal epithelial TJ proteins is unknown.

The present study was designed to determine whether depletion of TJ proteins is mediated by retained uremic toxins/metabolites and, if so, whether they can be removed by dialysis. To this end, we examined the effects of plasma taken from healthy controls and end-stage renal disease (ESRD) patients immediately before and after hemodialysis on the structure and function of the TJ in a polarized T84 human colonic epithelial cell line. When seeded on Transwell plates, T84 cells form a polarized impermeable monolayer that possesses many of the functional characteristics of the intestinal epithelium in vivo, including vectorial solute transport and barrier function $[14,15]$.

\section{Methods}

\section{Uremic and Control Plasma Preparations}

Five stable patients with ESRD maintained on hemodialysis for more than 3 months were recruited to provide blood samples before and immediately after hemodialysis treatment. A group of 5 healthy control individuals served as controls. Blood samples were collected in the heparinized tubes and plasma was immediately separated and stored at $-70^{\circ} \mathrm{C}$ until used. Plasma concentration of creatinine was measure by an autoanalyzer at the UC Irvine Medical Center's clinical laboratory. As expected, predialysis plasma creatinine levels were significantly elevated in the ESRD patients compared with the control group $(8.8 \pm 2.8 \mathrm{mg} / \mathrm{dl}$ vs. 0.8 $\pm 0.1, \mathrm{p}<0.0001)$. The study protocol was approved by the Institutional Review Board of the University of California Irvine (HS No. 2007-5572).

\section{Cell Culture and Incubation Studies}

T84 cells were obtained from American Type Culture Collection (Manassas, Va., USA) and were grown in DMEM/F12 medium (Invitrogen Inc., Carlsbad, Calif., USA) containing sodium bicarbonate $1.2 \mathrm{~g} / \mathrm{l}$, L-glutamine $2.5 \mathrm{mM}$, HEPES $15 \mathrm{mM}$, and sodium pyruvate $0.5 \mathrm{~mm}$ (Invitrogen) supplemented with $10 \%$ fetal calf serum. To form a polarized monolayer, cells were seeded in 12-well or 6-well plates with Transwell inserts (Fisher Scientific, Pittsburgh, Pa., USA) at 60,000 cells $/ \mathrm{cm}^{2}$. The cells were then used for measurement of TER and Western blot analyses. Cells cultured in Lab-Tek II Chamber Slides (Fisher Scientific) were used for immunofluorescence microscopy. The T84 monolayers were maintained for 21 days in complete medium. The medium was changed every other day and transepithelial electrical resistance (TER) was measured regularly using a Millicell ERS-2 Meter (EMD Millipore Inc., Billerica, Mass., USA). When a TER exceeding $1,000 \mathrm{~m} \Omega / \mathrm{cm}^{2}$ was reached, the monolayers were incubated for $24 \mathrm{~h}$ in DMEM/F12 medium containing 10\% plasma from either the healthy control individuals or ESRD patients before or after a 3-hour hemodialysis treatment. At the conclusion of the 24-hour incubation period, the TER was measured and cells were processed for immunostaining and confocal microscopy, as well as Western blot analysis.

Western Blot Analyses

The cells were lysed with M-PER Mammalian Protein Extraction Reagent (Fisher Scientific). Protein concentration in the cell lysate was determined by BSA assay kit (Pierce, Rockford, Ill., USA) and $20 \mu \mathrm{g}$ of total protein from each sample were fractionated on $4-12 \%$ Bis-Tris gradient gel (Invitrogen) at $120 \mathrm{~V}$ for $2 \mathrm{~h}$ and transferred to a nitrocellulose membrane. The membrane was then incubated with rabbit anticlaudin-1 or rabbit antioccludin or mouse anti-ZO1 (Invitrogen, Carlsbad, Calif., USA) antibodies at 1:250 dilutions, and antiactin antibody (Sigma-Aldrich, St. Louis, Mo., USA) at 1:10,000 dilution overnight. The appro- 


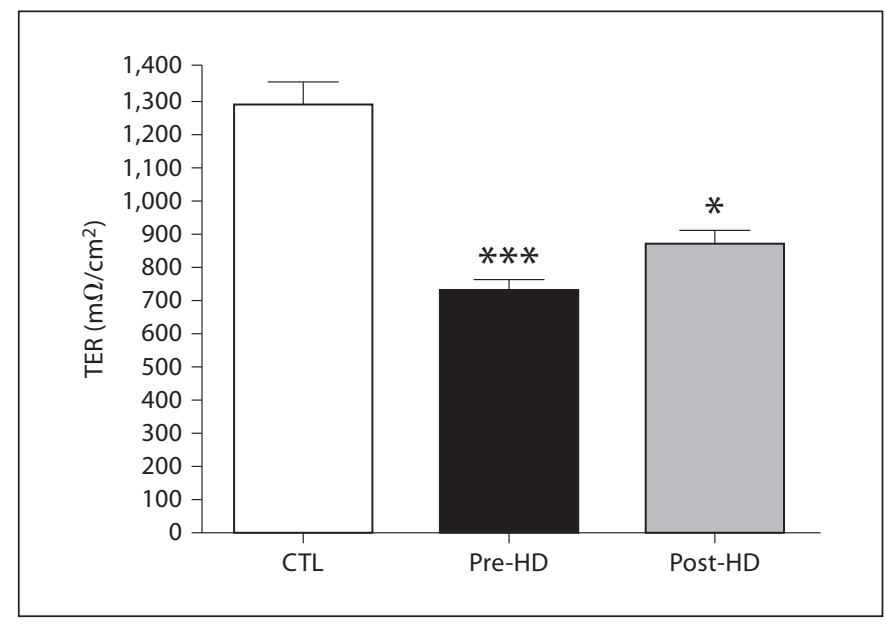

Fig. 1. Bar graph depicting TER in intestinal epithelial T84 cell monolayers exposed to media containing plasma obtained from healthy control (CTL) individuals and those containing prehemodialysis (Pre-HD) and posthemodialysis (Post-HD) plasma samples from ESRD patients. CTL vs. pre-HD, $\mathrm{p}=0.0003$; pre-HD vs. post-HD, $\mathrm{p}=0.027$.

priate horseradish peroxidase-conjugated secondary antibodies (Sigma-Aldrich, St. Louis, Mo., USA) were used at a 1:5,000 dilution. The membrane was visualized with SuperSignal West Pico (Pierce) and developed by autoluminography.

\section{Immunofluorescence Microscopy}

T84 cells were grown to confluence in chamber slides. The cells were washed with PBS and fixed with $3 \%$ paraformaldehyde. They were then incubated in solutions containing the primary antibodies against claudin-1 or ZO1 (Invitrogen) at 1:50 dilutions overnight at $4^{\circ} \mathrm{C}$. The cells were subsequently incubated in a solution containing the fluorescein isothiocyanate (AF555-R or AF488-FITC)-conjugated goat anti-rabbit IgG antibody (Invitrogen) for $90 \mathrm{~min}$ at 1:250 dilutions. After being mounted with FITC-compatible media, the cells were examined with a LSM 700 Confocal Microscope (Zeiss Inc., Oberkochen, Germany).

\section{Data Analysis}

Analysis of variance and Student's t test were used for statistical evaluation of the data, which are presented as means \pm SD. $\mathrm{p}<0.05$ was considered significant.

\section{Results}

\section{TER Data}

By design, the TER in T84 cells seeded on Transwell plates was monitored weekly and the incubation experiments were conducted when electrical resistance exceeding $1,000 \mathrm{~m} \Omega$ was reached to ensure full polarization and $\mathrm{TJ}$ formation. Incubation of epithelial monolayers in the media containing predialysis plasma from the ESRD group for $24 \mathrm{~h}$ resulted in a dramatic fall in transepithelial resistance when compared to those incubated in media containing plasma from the healthy control subjects. The magnitude of fall in TER in the epithelial monolayer exposed to media containing the ESRD patients' postdialysis plasma was significantly less than that observed with the predialysis plasma. However, dialysis did not fully reverse the effect of the uremic milieu on the TER (fig. 1).

\section{TJ Protein Data}

Incubation of the epithelial monolayers in media containing predialysis plasma from the ESRD group resulted in a marked reduction in $\mathrm{ZO} 1$ protein abundance and a near complete depletion of claudin-1 when compared to monolayers exposed to the media containing plasma obtained from the healthy control group. The effect of uremic plasma on occludin abundance was less severe than that seen with ZO1 and claudin-1 expression (fig. 2). Comparison of data obtained in epithelial monolayers exposed to media containing postdialysis with those incubated in media containing predialysis plasma samples from the ESRD group showed a significantly less severe depletion in ZO1, claudin-1, and occludin protein abundance (fig. 3). The immunohistological studies confirmed the data obtained by Western blot analysis described above. Representative immunohistological images obtained using fluorescein-tagged $\mathrm{ZO} 1$ and occludin antibodies in the monolayers incubated in media containing plasma from healthy controls and predialysis and postdialysis plasma from ESRD patients are shown in figure 4.

\section{Discussion}

The present study showed that exposure to plasma of an ESRD patient resulted in a significant reduction of ZO1, a near complete depletion of claudin-1, and a modest reduction of occludin in human colonic epithelial cells. The observed depletion of the key protein constituents of the epithelial TJ was accompanied by a marked fall in TER which denotes epithelial barrier dysfunction and increased permeability. These findings point to the presence of as-yet unidentified product(s) in the uremic milieu which are capable of damaging the structure and function of the gut's epithelial TJ. In an attempt to discern whether these unidentified product(s) are dialyzable, we compared the effects of plasma samples obtained immediately after hemodialysis treatment with those collected shortly before dialysis. The experiment revealed a 


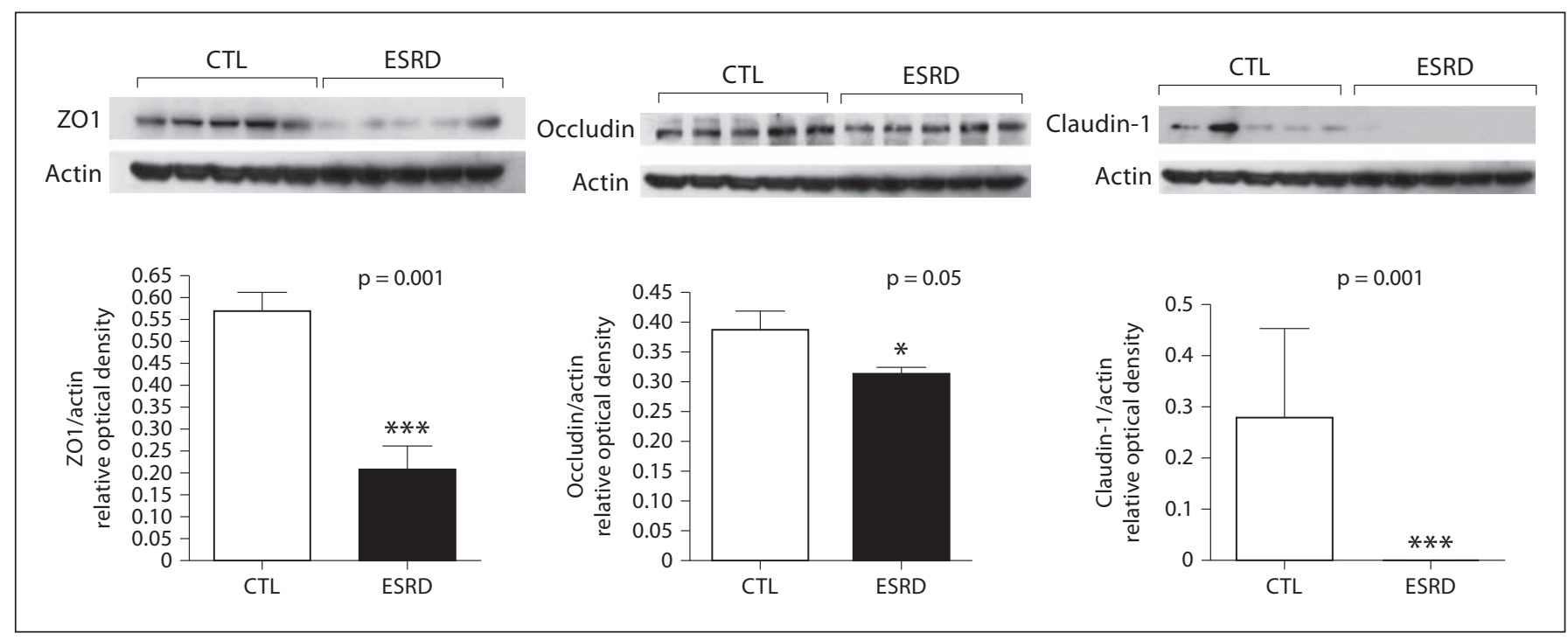

Fig. 2. Representative Western blots and group data depicting protein abundance of ZO1, occludin, and claudin-1 in intestinal epithelial T84 cell monolayers exposed to media containing plasma obtained from healthy control (CTL) individuals and those containing prehemodialysis plasma samples from ESRD patients.

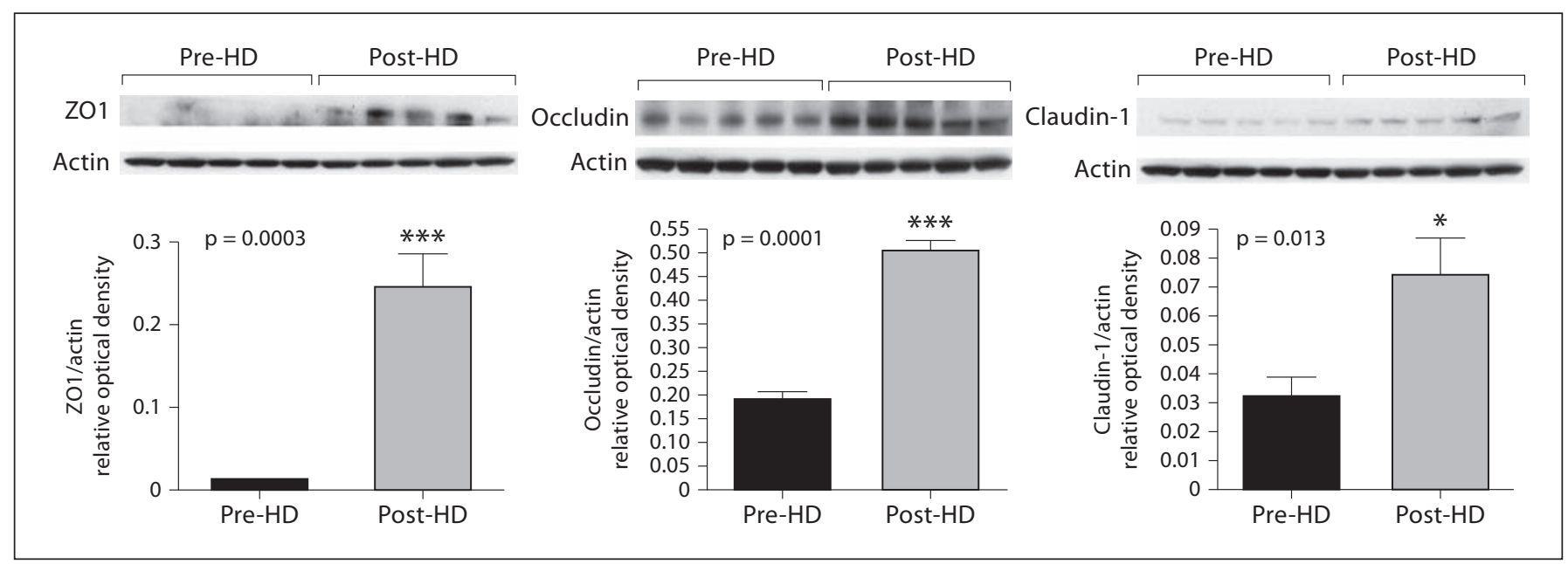

Fig. 3. Representative Western blots and group data depicting protein abundance of ZO1, occludin, and claudin-1 in intestinal epithelial T84 cell monolayers exposed to media containing plasma obtained from ESRD patients immediately before (Pre-HD) and after hemodialysis (Post-HD).

significantly less severe decline in TER and a lower depletion of TJ proteins in cells exposed to the ESRD patients' plasma obtained after dialysis than in cells exposed to plasma obtained before dialysis. While reducing the severity of the damaging effect of uremic milieu on the epithelial barrier function and TJ apparatus, hemodialysis did not fully reverse it. These effects appear to be mediated 'in part' by as-yet unidentified but partially dialyz- able products. Further studies are planned to identify the nature and the mechanism of action of the retained uremic products that promote intestinal barrier dysfunction and TJ destruction.

The results of the present in vitro experiments demonstrating the ability of plasma from an ESRD patient plasma to disrupt the barrier structure and function in cultured human enterocytes recapitulate the findings of our 
Fig. 4. Representative photomicrographs depicting claudin-1 and ZO1 immunostaining of intestinal epithelial T84 cell monolayers exposed to media containing plasma samples obtained from a healthy control individual and an ESRD patient immediately before and after hemodialysis and visualized by confocal microscopy.

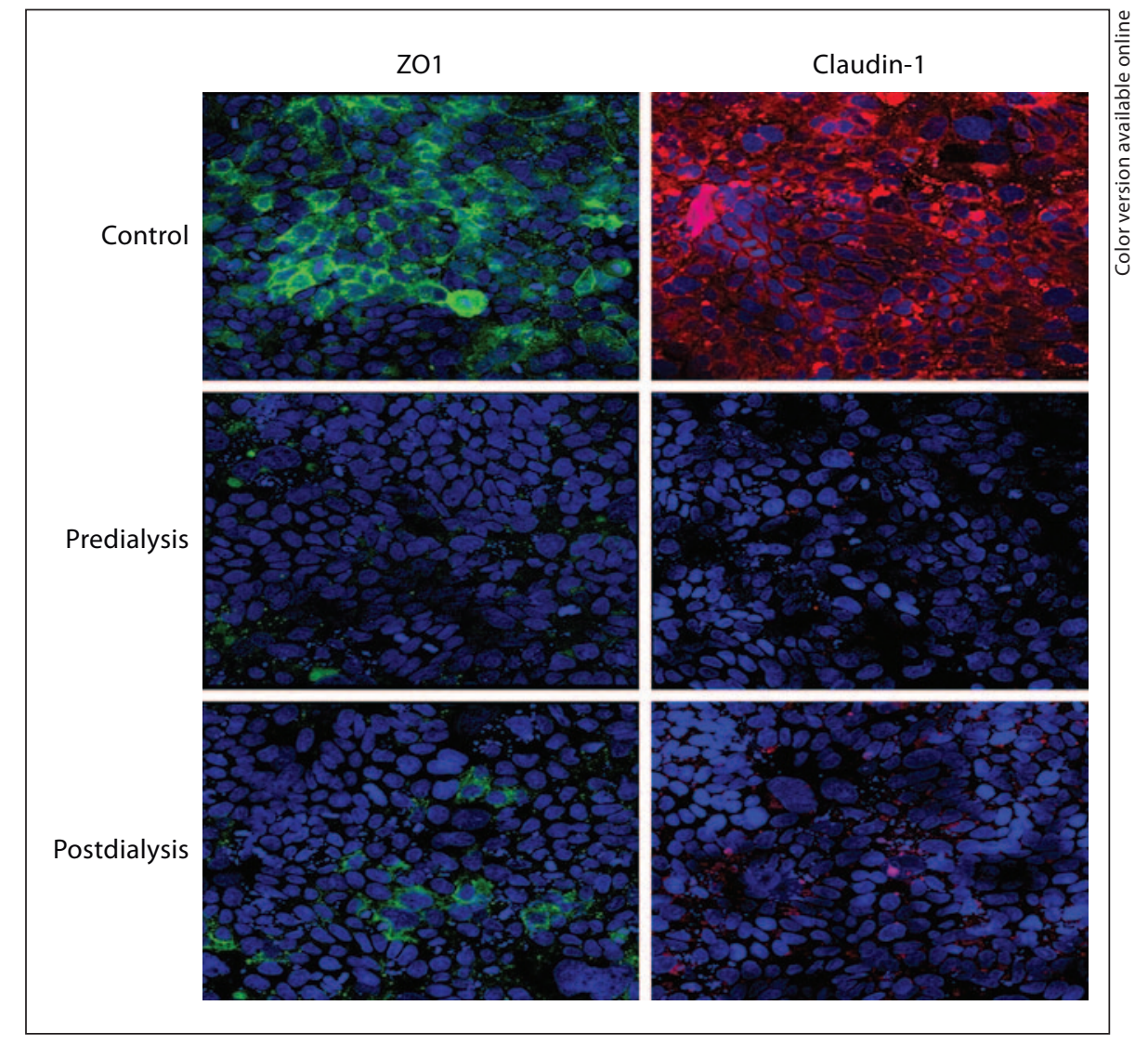

earlier studies which demonstrated degradation of the colonic epithelial TJ in two different animal models of CKD [13]. Together, these findings suggest that intestinal epithelial barrier structure and function must be similarly affected by the uremic milieu in patients with advanced CKD. This phenomenon can account for the presence of endotoxemia and increased intestinal permeability to high-molecular-weight polyethylene glycol in the patients with advanced CKD $[5-8,10]$. Paracellular influx of endotoxin and other noxious luminal products into the internal milieu through the damaged TJ apparatus can clearly contribute to the systemic inflammation and its adverse consequences in patients with CKD. In fact, Feroze et al. [5] recently demonstrated a direct association between severity of systemic inflammation and plasma endotoxin levels in the ESRD population. Identification of the retained uremic toxins/metabolites or other agents involved in this process and strategies to mitigate their impact could be critical in the management of the CKD-associated inflammation and its adverse consequences.

In addition to damaging the intestinal epithelial $\mathrm{TJ}$ and barrier function, uremia has recently been shown to result in profound alteration of the gut's microbial flora [16], which can further contribute to the systemic inflammation and uremic toxicity by generating harmful products $[17,18]$.

In conclusion, exposure to uremic milieu damages the intestinal epithelial TJ and impairs its barrier function. These effects are mediated by retained uremic toxin(s)/ metabolite(s) or other agents which are partially removed by hemodialysis.

\section{Acknowledgements}

This work was, in part, supported by Public Health Service Grant AI083663 (M.R.), NIH Grant DK082130 (H.M.), and grants from the National Institute of Diabetes, Digestive and Kidney Disease of the National Institutes of Health R01-DK078106, K24 DK091419-01, and a philanthropist grant from Mr. Harold Simmons.

\section{Disclosure Statement}

The authors have no conflicts of interest to declare. 


\section{References}

$>1$ Cachofeiro V, Goicochea M, de Vinuesa SG, Oubiña P, Lahera V, Luño J: Oxidative stress and inflammation, a link between chronic kidney disease and cardiovascular disease. Kidney Int Suppl 2008:S4-S9.

$>2$ Stenvinkel P: Inflammation in end-stage renal disease: the hidden enemy. Nephrology (Carlton) 2006;11:36-41.

-3 Turner JR: Intestinal mucosal barrier function in health and disease. Nat Rev Immunol 2009;9:799-809.

-4 Nusrat A, Turner JR, Madara JL: Molecular physiology and pathophysiology of tight junctions. IV. Regulation of tight junctions by extracellular stimuli: nutrients, cytokines, and immune cells. Am J Physiol Gastrointest Liver Physiol 2000;279:G851-G857.

$\checkmark 5$ Feroze U, Kalantar-Zadeh K, Sterling KA, Molnar MZ, Noori N, Benner D, Shah V, Dwivedi R, Becker K, Kovesdy CP, Raj DS: Examining associations of circulating endotoxin with nutritional status, inflammation, and mortality in hemodialysis patients. J Ren Nutr 2012;22:317-326.

6 Gonçalves S, Pecoits-Filho R, Perreto S, et al: Associations between renal function, volume status and endotoxaemia in chronic kidney disease patients. Nephrol Dial Transplant 2006;21:2788-2794.
7 Szeto CC, Kwan BC, Chow KM, et al: Endotoxemia is related to systemic inflammation and atherosclerosis in peritoneal dialysis patients. Clin J Am Soc Nephrol 2008;3:431436.

$>8$ Raj DS, Carrero JJ, Shah VO, Qureshi AR, Bárány $\mathrm{P}$, Heimbürger $\mathrm{O}$, Lindholm $\mathrm{B}$, Ferguson J, Moseley PL, Stenvinkel P: Soluble CD14 levels, interleukin 6, and mortality among prevalent hemodialysis patients. Am J Kidney Dis 2009;54:1072-1080.

$>9$ Magnusson M, Magnusson KE, Sundqvist T, Denneberg $\mathrm{T}$ : Increased intestinal permeability to differently sized polyethylene glycols in uremic rats: effects of low- and highprotein diets. Nephron 1990;56:306-311.

10 Magnusson M, Magnusson KE, Sundqvist T, et al: Impaired intestinal barrier function measured by differently sized polyethylene glycols in patients with chronic renal failure. Gut 1991;32:754-759.

11 de Almeida Duarte JB, de Aguilar-Nascimento JE, Nascimento $M$, et al: Bacterial translocation in experimental uremia. Urol Res 2004;32:266-270.

12 Vaziri ND, Dure-Smith B, Miller R, et al: Pathology of gastrointestinal tract in chronic hemodialysis patients: an autopsy study of 78 cases. Am J Gastroenterol 1985;80:608-611.
13 Vaziri ND, Yuan J, Rahimi A, Ni Z, Said H, Subramanian VS: Disintegration of colonic epithelial tight junction in uremia: a likely cause of CKD-associated inflammation. Nephrol Dial Transplant 2012;27:26862693.

14 Madara JL, Dharmsathaphorn K: Occluding junction structure-function relationships in a cultured epithelial monolayer. J Cell Biol 1985;101:2124-2133.

-15 Dharmsathaphorn K, McRoberts JA, Mandel KG, Tisdale LD, Masui H: A human colonic tumor cell line that maintains vectoral electrolyte transport. Am J Physiol 1984; 246:G204-G208.

16 Vaziri ND, Wong J, Pahl MV, Piceno YM, Yuan J, DeSantis TZ, Ni Z, Nguyen TH, Andersen GL: Chronic kidney disease alters the composition of intestinal microbial flora. Kidney Int 2012, E-pub ahead of print.

17 Vaziri ND: CKD impairs barrier function and alters microbial flora of the intestine - a major link to inflammation and uremic toxicity. Curr Opin Nephrol Hypertens 2012, Epub ahead of print.

18 Aronov PA, Luo FJ, Plummer NS, Quan Z, Holmes S, Hostetter TH, Meyer TW: Colonic contribution to uremic solutes. J Am Soc Nephrol 2011;22:1769-1776. 